\title{
John Perry's neglected critique of Kelvin's age for the Earth: A missed opportunity in geodynamics
}

\begin{abstract}
Philip England, Department of Earth Sciences, Oxford University, Parks Road, Oxford OX1 3PR, UK; Peter Molnar, Department of Geological Sciences and Cooperative Institute for Research in Environmental Sciences, University of Colorado, Boulder, Colorado 80309, USA; Frank Richter, Department of the Geophysical Sciences, 5734 S. Ellis Ave. Chicago, Illinois 60637, USA
\end{abstract}

\begin{abstract}
Many readers know the tale of how William Thomson (later Lord Kelvin) calculated the age of the Earth from physical principles and adhered for over 50 years to an estimate that was far younger than geologists' estimates, despite the virtually unanimous opposition of the geological community of the time. The prevalent version of this tale alleges that the discovery of radioactivity simultaneously provided the demonstration (through radiometric dating) that Kelvin had greatly underestimated the age of the Earth and the explanation of why he was wrong (radioactivity being a source of heat that invalidated Kelvin's calculation). We show this popular story to be incorrect; introducing the known distribution of radioactivity into Kelvin's calculation does not invalidate its conclusion. In 1895, before the discovery of radioactivity, John Perry showed that convection in the Earth's interior would invalidate Kelvin's estimate for the age of the Earth, but Perry's analysis was neglected or forgotten, with the consequence that a powerful argument in favor of mobilism was overlooked during the first few decades of debate about continental drift.
\end{abstract}

\section{INTRODUCTION}

The story of Kelvin and the age of the Earth is often told as a David-and-Goliath struggle, with the geologists in the role of the underdog armed only with the slender sword of geological reasoning, while Lord Kelvin bludgeoned them with the full force and prestige of mathematical physics. Kelvin's comeuppance is often taken as evidence that simple physics ought not to be applied to geological problems, but there have been numerous occasions when simple physical models have had great explanatory power in geology. Perry's critique of Kelvin's calculation reminds us that even well-posed physical models can sometimes be misleading, but recognition of their flaws may lead to major advances.

\section{KELVIN'S CALCULATION OF THE AGE OF THE EARTH}

We cannot, in this short space, approach a full description of Kelvin's arguments about the age of the Earth. The reader is referred to Burchfield (1975) for a detailed account of the controversy, to Lindley (2004) for a very readable biography, with an insightful account of the debate on the age of the Earth in the context of Kelvin's other work, and to Richter (1986) and
Stacey (2000) for other geophysical perspectives on Kelvin's calculations.

Fourier laid the groundwork for the mathematical analysis of the flow of heat in his treatise Théorie Analytique de la Chaleur (Fourier, 1822), and he made arguments that the Earth must be cooling (Fourier, 1827), with which Lyell was certainly familiar (Lyell, 1830, p. 140-141). Kelvin first wrote on heat when he was 16, clarifying some of Fourier's mathematics, and he first addressed the age of the Earth in 1844 when he showed that, if one were to assume that the Earth is a solid body cooling from an initially high temperature, measurement of the rate of heat loss from its surface would place bounds on its age.

Kelvin imagined the Earth to have solidified from an originally molten state, such that its initial condition was of uniform temperature, $T_{0}$, with its surface maintained at a constant temperature for all time. Under these assumptions, temperature depends upon depth, $z$, below the Earth's surface, and upon time, $t$, since the initial state. Fourier had shown that the diffusion of heat in a solid is described (in one spatial dimension and in the absence of heat sources) by

$$
\frac{\partial T}{\partial t}=\kappa \frac{\partial^{2} T}{\partial z^{2}}
$$

where $T$ is temperature, and $\kappa$ is the thermal diffusivity (Table 1 ).

We take the surface of the Earth $(z=0)$ to be at a temperature of $0{ }^{\circ} \mathrm{C}$; given that any plausible estimate for $T_{0}$ is several thousand such degrees, the small deviations of the Earth's surface temperature from $0{ }^{\circ} \mathrm{C}$ may be neglected. With the stated initial and surface conditions, and with the condition that the temperature tends to $T_{0}$ at infinite depth, the solution to Equation 1 is

$$
T(x, t)=T_{0} \operatorname{erf}\left(\frac{z}{2 \sqrt{\kappa t}}\right),
$$

where $\operatorname{erf}(x)$ is the error function (e.g., Carslaw and Jaeger, 1959). The temperature gradient at the Earth's surface is

$$
G=\left.\frac{\partial T}{\partial z}\right|_{z=0}=\frac{T_{0}}{\sqrt{\pi \kappa t}}
$$

(Fourier, 1822; Kelvin, 1863a). An excellent introduction to thermal diffusion is given by Carslaw and Jaeger (1959), and all solutions to the diffusion problems we discuss can be found there.

The expressions of Equations 2 and 3 are familiar to many as the solution for the cooling of the oceanic lithosphere when it is treated as a half-space (Turcotte and Oxburgh, 1967; Parsons and Sclater, 1977; see also Turcotte and Schubert, 2002, p. 157). This problem is mathematically identical to Kelvin's problem, though the age involved is that of the ocean floor, rather than of the Earth.

Equations 2 and 3 express the fact that, in a given time, $t$, the average distance that heat can diffuse is approximately $\sqrt{\pi \kappa t}$ 
and, in consequence, at any time, $t$, material at a depth greater than $\sqrt{\pi \kappa t}$ is still at its original temperature and, to a good approximation, the temperature gradient between the surface and the depth is $T_{0} / \sqrt{\pi \kappa t}$.

The key point of Kelvin's model is that Equation 3 may be inverted to give $t$, the age of the Earth, in terms of the geothermal gradient, $G$, or the heat flux, $Q$, observed at the Earth's surface now:

$$
t=\frac{\left(T_{0} / G\right)^{2}}{\pi \kappa}=\frac{\left(K T_{0} / Q\right)^{2}}{\pi \kappa} .
$$

When Kelvin first made these arguments in 1844 and 1846, geothermal data were not available to him. By the time he returned to the problem 15 years later, geothermal gradients had been measured in several parts of the world. Kelvin (1863a) quoted measurements of between $1 / 110^{\circ} \mathrm{F}$ and $1 / 15^{\circ} \mathrm{F}$ of temperature increase per foot of depth and chose as a mean gradient for his calculation $1 / 50{ }^{\circ} \mathrm{F}$ per foot (or $\sim 36{ }^{\circ} \mathrm{C} / \mathrm{km}$ ). With an assumption of the initial temperature, $T_{0}$, based on melting experiments on rocks $\left(7000^{\circ} \mathrm{F}, \sim 3900{ }^{\circ} \mathrm{C}\right)$ and with an estimate, based on laboratory measurements, of $\kappa \approx 1.2 \times 10^{-6}$ $\mathrm{m}^{2} \mathrm{~s}^{-1}$, this gradient yields an age of $96 \mathrm{Ma}$; Kelvin (1863a) gave bounds of $24 \mathrm{Ma}$ and $400 \mathrm{Ma}$ on the age to take account of uncertainties in thermal gradient and thermal conductivity.

For the rest of our discussion, we shall use heat flux, rather than thermal gradient, because it is the more fundamental unit, in part because it is unaffected by near-surface contrasts in con-

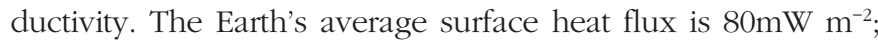
the average for the continents is $\sim 60 \mathrm{~mW} \mathrm{~m}^{-2}$ and, except in the shields, the background heat flux in the continents (after the contribution from near-surface radioactivity has been stripped out) is $\sim 40 \mathrm{~mW} \mathrm{~m}^{-2}$ (Sclater et al., 1981). We therefore take the range of surface heat flux that has to be matched by any proposed thermal history of the Earth to be $40-80 \mathrm{~mW} \mathrm{~m}^{-2}$. Using this range, and the values of other quantities given in Table 1, the modern equivalent of Kelvin's estimate is 24-96 Ma.

Scientists derive an extra measure of confidence in a conclusion if they can arrive at it by more than one independent route, and this was no doubt true in Kelvin's case. He had shown that, given what was known at the time, the only plau-

TABLE 1. NOTATION FOR, AND ASSUMED VALUES AND UNITS OF, RELEVANT PHYSICAL QUANTITIES

\begin{tabular}{llcc}
\hline \hline$A$ & Heat production & & $\mu \mathrm{W} \mathrm{m}{ }^{-3}$ \\
$c$ & Specific heat & $10^{3}$ & $\mathrm{~J} \mathrm{~kg}^{-1} \mathrm{~K}^{-1}$ \\
$G$ & Geothermal gradient at the Earth's surface & & ${ }^{\circ} \mathrm{C} / \mathrm{km}^{-1}$ \\
$K$ & Thermal conductivity & 3 & $\mathrm{~W} \mathrm{~m}^{-1} \mathrm{~K}^{-1}$ \\
$\kappa$ & Thermal diffusivity $(=K / \rho c)$ & $10^{-6}$ & $\mathrm{~m}^{2} \mathrm{~s}^{-1}$ \\
$L$ & Conducting lid to the Earth & & $\mathrm{m}$ \\
$Q$ & Surface heat flux & $40-80$ & $\mathrm{~mW} \mathrm{~m}^{-2}$ \\
$R$ & Radius of the Earth & $6.4 \times 10^{6}$ & $\mathrm{~m}$ \\
$\rho$ & Density & 3300 & $\mathrm{~kg} \mathrm{~m}^{-3}$ \\
$t$ & Time & & $\mathrm{S}$ \\
$T$ & Temperature & 1300 & ${ }^{\circ} \mathrm{C}$ \\
$T_{0}$ & Initial temperature of the Earth & ${ }^{\circ} \mathrm{C}$ \\
$T_{i}$ & Interior temperature of the Earth (Equation 5) & & $\mathrm{m}$ \\
$z$ & Depth & & \\
\hline
\end{tabular}

GSA TODAY, JANUARY 2007 sible source for the energy radiated by the sun was internal energy derived from gravitational potential energy release during its accretion. He had calculated the amount of this energy and concluded that it could sustain the present rate of radiation from the sun for no more than 100 m.y. (Stacey, 2000). The agreement between these two apparently independent estimates strengthened Kelvin's conviction in his calculation of the age of the Earth.

\section{KELVIN AND THE GEOLOGISTS}

The early nineteenth-century formulation of Uniformitarianism was commonly expressed through Hutton's aphorism, "No vestige of a beginning, no prospect of an end." The doctrine that the Earth was of unlimited age allowed geologists to explain any phenomenon not by the laws of physics, but by "reckless drafts on the bank of time" (Chamberlin, 1899). For Kelvin, this game without rules was simply not scientific; indeed, it was forbidden by the laws of thermodynamics, which he had played such a large part in developing.

In 1867, Kelvin had a conversation with the geologist Andrew Ramsay, "almost every word of which remains stamped on my mind to this day" (Kelvin, 1899; see also Lindley, 2004, p. 175-177). They had been listening to Archibald Geikie discussing the

... geological history of the actions by which the existing scenery of Scotland was produced. I asked Ramsay how long a time he allowed for that history. He answered that he could suggest no limit to it. I said "You don't suppose geological history has run through 1,000,000,000 years?" "Certainly I do." "10,000,000,000 years?" "Yes." "The sun is a finite body. You can tell how many tons it is. Do you think it has been shining for a million million years?" "I am as incapable of estimating and understanding the reasons which you physicists have for limiting geological time as you are incapable of understanding the geological reasons for our unlimited estimates." I answered, "You can understand the physicists' reasoning perfectly if you give your mind to it."

It is easy to overlook the enormous gains to geology that came simply from having to fight the battle with Kelvin about the age of the Earth. By the end of the nineteenth century, the doctrine of a steady-state Earth of indefinite age had been replaced by the understanding that present geological processes provide guides to, and constraints upon, past geological events: "No vestige of a beginning, no prospect of an end" had been replaced by "The present is the key to the past" (Chamberlin, 1899; Burchfield, 1975). Even before radioactivity was discovered, geologists had come to accept that the age of the Earth was finite and that estimating the age by quantitative reasoning was a crucial part of geological endeavor.

What nobody did until 1895, however, was to put their mind, as Kelvin had suggested, to the physicists' reasoning, and discover the flaw in it.

\section{KELVIN'S ASSUMPTIONS}

A single principle underlies all Kelvin's arguments about the age of the Earth: that energy is conserved. To carry out his analyses, Kelvin added three assumptions. Two assumptions applied only to his arguments about the Earth: that the Earth 
was rigid and that its physical properties were homogeneous. The third assumption, that there was no undiscovered source of energy, applied both to the Earth and to the sun.

The conventional story alleges that his third assumption was Kelvin's undoing, but as we shall show below, this story, while correct for the age of the sun, is incorrect for the age of the Earth. The real flaw in Kelvin's argument about the Earth was pointed out by one of his former assistants, John Perry, in 1895-a year before radioactivity was discovered and eight years before it became recognized as a source of heat.

Perry (1895a) wrote: "I have sometimes been asked by friends interested in geology to criticise Lord Kelvin's calculation of the probable age of the Earth. I have usually said that it is hopeless to expect that Lord Kelvin should have made an error in calculation." Instead of focusing on Kelvin's calculations, Perry suggested, one should examine his assumptions. In Kelvin's model, the present supply of heat to the Earth's surface is derived from the cooling of a shallow outer layer of thickness, $\sqrt{\pi \kappa t}$ (Equation 3 ). If, however, the thermal conductivity inside the Earth were much higher than at the surface, then the deep interior would also cool, providing a large store of energy to maintain the surface heat flux. In that case, Kelvin's estimate of the age of the Earth would be too low, potentially by a large multiple.

Perry had two reasons for postulating a higher conductivity in the interior. First, experimental evidence showed an increase, if modest, in conductivity of rocks with temperature; in addition, the Earth's increase in density with depth implies a greater proportion of iron and other materials that conduct heat better than do silicates. More radically, he argued (Perry, 1895a) that convection in the fluid, or partly fluid, interior of the Earth would transfer heat much more effectively than would conduction: "... much internal fluidity would practically mean infinite conductivity for our purpose."

Unable to calculate the role of convection in a complete fashion, Perry proposed approximating its effect by a high "quasi-conductivity" in the interior of the Earth. He suggested a simple thought experiment to illustrate this point. Suppose that only a thin outer skin of the Earth, of thickness $L$, transfers heat by conduction, and that the rest of the Earth has effectively perfect thermal conductivity. The heat flux through the conducting lid at any time, $t$, will be $K T_{i} / L$, where $T_{i}$ is the interior temperature; this flux, multiplied by the Earth's surface area, will equal the rate at which the interior is losing heat:

$$
4 \pi R^{2} \frac{K T_{i}}{L}=\frac{4 \pi R^{3}}{3} \rho c \frac{d T_{i}}{d t} .
$$

The solution for $T_{i}$ is

$$
T_{i}=T_{0} \exp \left(\frac{-3 \kappa t}{R L}\right)
$$

(Perry 1895a, p. 255, footnote 1). For a lid of thickness $L=$ $100(50) \mathrm{km}$, the heat flux given by Equation 6 decays with a time constant $(R L / 3 \kappa)$ of $6(3) \mathrm{Ga}$, and the Earth's measured heat flux is consistent with any age up to $2 \mathrm{Ga}$ (Fig. 1A).

A less-than-perfect thermal conductivity for the interior of the Earth would cause it to cool down more slowly than is suggested by Equation 6. Perry and Heaviside modified Kelvin's calculation for the case of large, but finite, interior
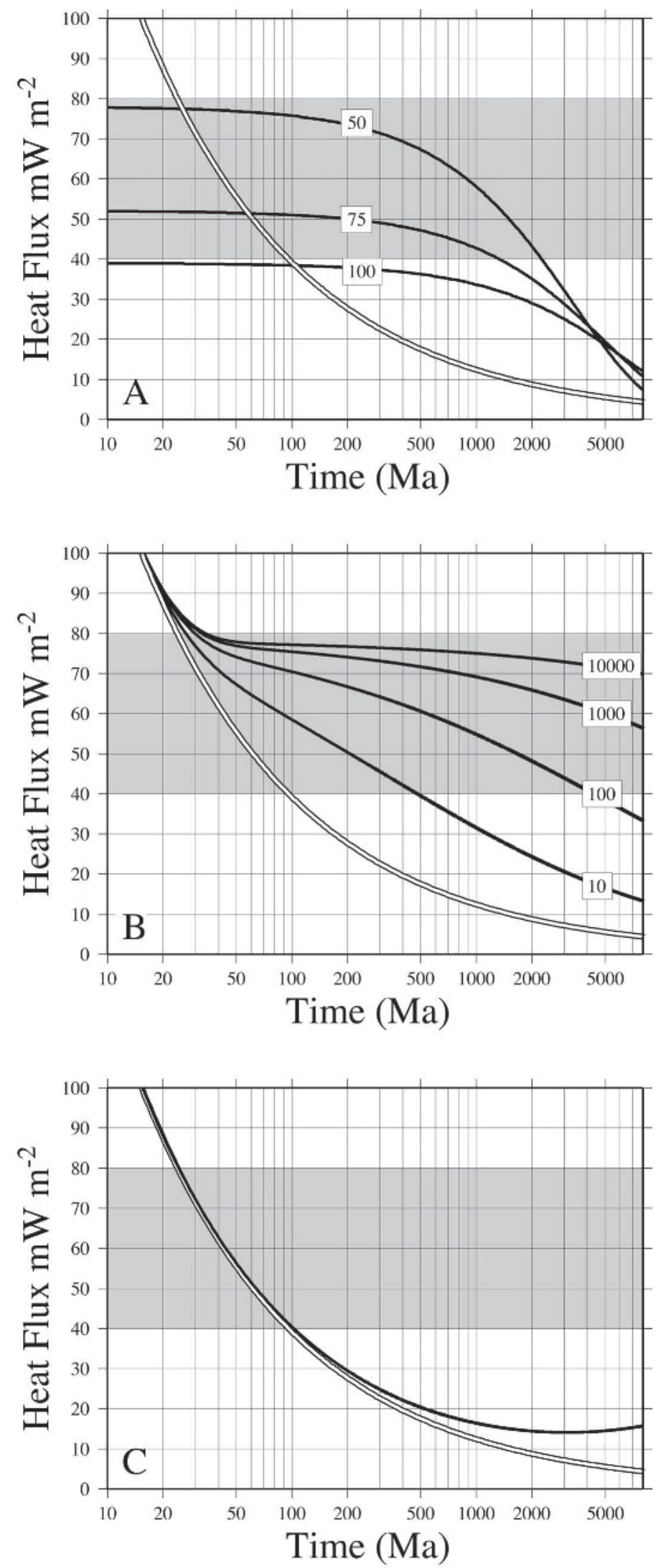
conductivity (Perry, 1895b; Carslaw and Jaeger, 1959, p. 322) and showed that the Earth's present heat flux is consistent with an age of gigayears, provided that the conducting lid is a few tens of kilometers thick and the effective (or "quasi-") conductivity of the interior is $\sim 100$ times greater than that of the lid (Fig. 1B).

In modern parlance, Perry's "quasi-conductivity" for a convecting fluid would be expressed by the Nusselt number, which is the ratio of the heat flux out of a convecting layer to the heat that would be carried across the same layer by conduction alone. For the conditions appropriate to the Earth's mantle, this ratio is likely to be in the range of 30-100 (McKenzie et al., 1974; Turcotte and Oxburgh, 1967). In the past 30 years, numerous studies of the Earth's thermal history, using more rigorous parameterizations of convection than were available to Perry, have confirmed that the surface heat flux provides, at best, a weak constraint on the age of the Earth. Indeed, as Richter (1986) points out, the present challenge-given the age of the Earth-is to determine what physical conditions in its interior lead to the present value of the heat flux.

\section{RADIOACTIVITY}

Curie and Laborde (1903) demonstrated that radioactive decay releases heat, and several people soon argued that this source of heat was great enough to overturn Kelvin's conclusion about the age of the Earth. In 1904, Rutherford spoke on the matter at a meeting in the Royal Institution:

I came into the room, which was half dark, and presently spotted Lord Kelvin in the audience and realized that I was in for trouble at the last part of the speech dealing with the age of the Earth, where my views conflicted with his. To my relief he fell fast asleep but as I came to the important point, I saw the old bird sit up, open an eye and cock a baleful glance at me! Then sudden inspiration came, and I said Lord Kelvin had limited

Figure 1. Calculations of the surface heat flux of the Earth according to the models discussed in the text. Shaded band in each panel of this figure shows the range of estimates for the mean surface heat flux at present day (see text). Double line shows the surface heat flux out of a semi-infinite medium of thermal diffusivity $10^{-6} \mathrm{~m}^{2} \mathrm{~s}^{-1}$, whose initial temperature is constant at $1300{ }^{\circ} \mathrm{C}$ (Kelvin's calculation: Equation 3, with the values of parameters given in Table 1). (A) The flux of heat through the surface of a sphere of radius $6400 \mathrm{~km}$, whose outer $50 \mathrm{~km}, 75 \mathrm{~km}$, or $100 \mathrm{~km}$ is a solid lid with thermal conductivity $3 \mathrm{~W} \mathrm{~m}^{-1} \mathrm{~K}^{-1}$, and whose interior is a well-stirred fluid (Equation 5). This is Perry's thought experiment for the cooling of a convecting planet (Equation 6). (B) The surface heat flux out of a layer that is $50 \mathrm{~km}$ thick, has a thermal diffusivity $10^{-6} \mathrm{~m}^{2} \mathrm{~s}^{-1}$, and thermal conductivity $3 \mathrm{~W} \mathrm{~m}^{-1} \mathrm{~K}^{-1}$. This layer is underlain by a half-space whose thermal conductivity is 10, 100, 1000, and 10000 times greater than that of the top layer, with its other properties the same. The medium has initial temperature $T_{0}=1300{ }^{\circ} \mathrm{C}$, and its surface is maintained at zero temperature (Carslaw and Jaeger, 1959, p. 322). This is the modification to the model illustrated in Figure 1A that was suggested by Perry and Heaviside (Perry, 1895b). (C) The surface heat flux from a semi-infinite medium that is heated internally (Carslaw and Jaeger, 1959, p. 79). The thermal diffusivity is $10^{-6} \mathrm{~m}^{2} \mathrm{~s}^{-1}$, the initial temperature of the medium is $1300{ }^{\circ} \mathrm{C}$ throughout, the surface is maintained at zero temperature for all time, $t>0$, and the medium is heated internally at a rate $A_{0}=$ $0.02 \mu \mathrm{W} \mathrm{m}{ }^{-3}$. This calculation shows the negligible influence of the Earth's radioactive heat production upon Kelvin's calculation. the age of the Earth, provided no new source of heat was discovered. That prophetic utterance refers to what we are now considering tonight, radium! Behold! The old boy beamed at me. (Eve, 1939, p. 107).

Heat given out by radium obviously cannot be the missing energy, because the half-life of its dominant isotope is 1600 yr, but it soon was demonstrated (through assuming equilibrium in their decay series) that uranium and thorium provide heat sources with half-lives of gigayears. Within a few years, radiometric dating of rocks had stretched the age of the Earth to 2 Ga (Dalrymple, 1991, p. 69-78). Measurements of heat production in crustal rocks permitted the interpretation that all the Earth's surface heat flux could be explained by heat generated in a layer of granite a few tens of kilometers thick, but that interpretation was not subjected to anything like the rigorous scrutiny given to Kelvin's argument. Bailey Willis, recording these times forty years on, wrote: "Thanks to Madame Curie, the inexhaustible energies of the atom of the globe ... are potentially available to geological speculation." (Willis, 1942; Oreskes, 1999, p. 48-51). In other words, Chamberlin's "reckless drafts" were now on the bank of heat, rather than on the bank of time.

We now know that the crust does not contain enough radioactive heat to explain the surface heat flux; nevertheless, it is still frequently stated that, because the discovery of radioactive heat undermined an assumption behind Kelvin's calculation, it also undermined his conclusion. This statement is logically incorrect; Kelvin's conclusion would be undermined by that discovery only if incorporation of the Earth's radioactive heat into his calculation produced a substantially different age for the Earth.

Modern estimates for the total present rate of radioactive heat generation within the Earth are $\sim 2 \times 10^{13} \mathrm{~W}$, equivalent to a surface heat flux of $40 \mathrm{~mW} \mathrm{~m}^{-2}$ (Sclater et al., 1981). It might, therefore, seem that the Earth's internal heat production can account for its surface heat flux, but we must recall that this heat production is distributed through the whole volume of the mantle, and diffusion of heat is slow. Kelvin's calculation (Equation 2) shows that only the outer $\sim 100 \mathrm{~km}$ of the Earth will cool by conduction in 100 m.y., and we should correspondingly expect that, if Kelvin's calculation were re-run with the inclusion of radioactivity, only the heat generated in the outermost part of the Earth would contribute to the surface heat flux.

The heat flux at the surface of a half-space, whose temperature is zero at time $t=0$ and that is thereafter heated internally at a rate $A$ per unit volume is

$$
Q=\frac{2 A}{\sqrt{\pi}} \sqrt{\kappa t}
$$

(Carsaw and Jaeger, p. 79). This expression shows that only the heat generated within a distance $\sim \sqrt{\kappa t}$ of the surface contributes to the surface heat flux at any time, $t$.

Kelvin's calculation can be adjusted to take into account internal heating by adding the heat flux from Equation 7 to that from Equation 3. If the total rate of radioactive heat generation $\left(2 \times 10^{13} \mathrm{~W}\right)$ is distributed evenly through the mantle, it is equivalent to a volumetric rate of $A=0.02 \mu \mathrm{W} \mathrm{m} \mathrm{m}^{-3}$. This level of heating raises the heat flux above that in Kelvin's calculation by 
a few $\mathrm{mW} \mathrm{m} \mathrm{m}^{-2}$ the first g.y. of Earth's history (Fig. 1C); consequently-even if Kelvin had included radioactive heat in his calculation-his estimate of the age of the Earth would have been unaffected (Richter, 1986).

Thus, the discovery of radioactivity did not invalidate Kelvin's calculation for the age of the Earth. In a rigid Earth, with or without radioactivity, heat is delivered to the surface by conduction through a shallow layer, which can maintain a rate of heat loss comparable to today's for only a small fraction of what we now know to be the Earth's age.

\section{WHY WERE PERRY'S ARGUMENTS FORGOTTEN, AND WHY WAS THE MYTH ACCEPTED?}

Perry argued that Kelvin's estimate of the age of the Earth could be many times too low if its interior were fluid with a high "quasi-conductivity" due to its convection. The suggestion of a fluid mantle was not new; indeed, it was widely understood as the necessary condition for isostasy. Perry published his case (Perry, 1895a, 1895b, 1895c) in the pages of Nature, which, judging by the passion that authors of papers attached to their agendas, was as prominent an organ of scientific discourse in 1895 as it is today. It therefore seems probable that those concerned with the debate about the age of the Earth would have known of Perry's argument. So why was the argument not accepted in the decade before radioactive heat became established or, indeed, thereafter?

Part of the explanation may be that the debate often descended to the use of rhetoric in place of scientific argument. Kelvin (1899) cites many examples of rhetoric from his opponents and, while Kelvin himself was generally quite measured in his replies, P.G. Tait (in his self-appointed role as Kelvin's bulldog) did not hesitate to respond in kind (Lindley, 2004, p. 175-178) (see also letters from Tait, quoted in Perry, 1895a). Faced with all this hot air, Mark Twain (1903) concluded, "As Lord Kelvin is the highest authority in science now living, I think we must yield to him and accept his view." Perhaps a parallel sentiment led to Kelvin's view being supplanted by Rutherford's, after 1904.

It is also probable that Perry was not understood by most people who cared about the age of the Earth. A thread running through much of Kelvin's writing on this subject is that the geological community of his time shied away from mathematics (Lindley, 2004). Geologists may have regarded Perry's exchanges with Kelvin (Kelvin, 1895; Perry, 1895a, 1895b, 1895c) as nothing more than an incomprehensible tussle among physicists. Furthermore, that tussle may have seemed irrelevant. As suggested by Andrew Ramsay's tart conversation with Kelvin, many geologists felt that Kelvin was incapable of understanding geological reasoning, so some of the resistance to his arguments (and indifference to Perry's refutation) may have stemmed from the belief that geology is too complex to be encapsulated in a mathematical model.

Kelvin knew, however, that simple models are an indispensable tool in science, whose purpose is to allow analysis of the major features of phenomena, not slavishly to reproduce all their details. Many of the most useful models are underlain by a principle famously expressed by Einstein: "Everything should be made as simple as possible, but not simpler."
It is worth illustrating the explanatory power of simple models in geology by a relevant modern example. Suppose we wished to explain the flux of heat from the ocean floor. A model that accounted accurately only for major, obvious, geological variables (different thermal properties and thicknesses of sediments, crustal layers, and mantle; hydrothermal circulation; off-ridge volcanism, etc.) would still have at least a dozen parameters, many of them poorly known, and would require millions of calculations (probably numerical) simply to explore the parameter space; consequently, its results would be impossible to grasp. In contrast, treating the ocean floor as a homogeneous conducting medium yields a simple analytical solution (Equation 2) that captures the main features of the cooling of the seafloor as it moves away from the oceanic ridges and explains the surface elevations of half of the Earth's surface (Parsons and Sclater, 1977; Turcotte and Oxburgh, 1967).

What Kelvin did not allow for is that, to varying degrees, all simple models are bound to fail, and we may learn as much by their failure as by their successes. The cooling half-space model fails for young ocean floor because of the influence of hydrothermal circulation; however, the degree to which heat flux departs from the simple model allows us to estimate the amount of heat transferred by that circulation, and hence the flux of water through the oceanic ridges (Lister, 1972; Morton and Sleep, 1985). The model also fails for ocean floor older than ca. 80-90 Ma but, in failing, reveals a second scale of convection in the mantle and allows us to estimate the thickness of the plates (Crosby et al., 2006; Parsons and McKenzie, 1978; Parsons and Sclater, 1977).

Perry's analysis of the failure of Kelvin's model similarly carried the strong implication that the solid Earth can carry heat by convection. Kelvin felt that he was on firm ground in rejecting the notion of a fluid interior to the Earth: he knew from the study of Earth tides that at least the outer $1500 \mathrm{~km}$ of the mantle is as rigid as steel (Kelvin, 1863b). Perry (1895a) tried hard to change Kelvin's mind on this point, using language specifically addressed to Kelvin's way of thinking:

... the real basis of your calculation is your assump-

tion that the solid earth cannot alter its shape ... even in 1000 million years, under the action of forces constantly tending to alter its shape, and yet we see the gradual closing up of passages in a mine, and we know that wrinkling and faults and other changes of shape are always going on in the earth under the action of longcontinued forces. I know that solid rock is not like cobbler's wax, but $10^{9}$ years is a long time, and the forces are great. (Perry, 1895a)

The reference to cobbler's wax is deliberate. Kelvin, like many others at the time, thought that light could not pass through a vacuum, but required a physical medium, the ether, for its propagation. That medium had to possess elastic properties at very short times, to allow light waves to propagate, but it needed to be weak at longer time scales, so that the Earth could move freely through it. Although he could not find a satisfactory mathematical formulation for the ether, Kelvin was fond of a physical demonstration that illustrated its required physical properties (Lindley, 2004, p. 247). He 
placed water in a glass cylinder, floated a layer of cork on the water, covered that with a layer of Scottish shoemaker's wax, and finally placed bullets on the top. Over a short period of observation, nothing visible happened, but after six months the corks and the bullets were within the wax, and after a year, the corks were on the top and the bullets were on the bottom. The wax exhibited strength on the short time scale, but was weak on the long time scale. These, qualitatively, were the required properties of the ether and-Perry was implying — of the Earth's mantle. Kelvin completely missed the point and so, it seems, did everyone else.

If Perry's analysis had been absorbed by the scientific community of the day, then the first radiometric ages for the Earth would have come as confirmation of the convective explanation for the Earth's surface heat flux, and the "fixist" view of the Earth, which exerted such a brake on geological progress in the first half of the twentieth century, would have been difficult to sustain. As it was, however, proponents of continental drift and convection needed repeatedly to make arguments in favor of a fluid Earth, against considerable skepticism (e.g., Oreskes, 1999). As late as the 1960s, geophysical models were being constructed that tried to match the surface heat flux employing a solid Earth with elaborate distributions of thermal conductivity and heat generation.

We are left with the question as to why the myth persists that the discovery of radioactivity simultaneously proved Kelvin wrong and provided the explanation for his error. Part of the answer, perhaps, is that it makes a good story. Rutherford's biographer (Eve, 1939) reports that he repeated his tale of thinking on his feet in front of the "old bird" Kelvin on many occasions; it is entirely possible that the pleasing form of the anecdote, and the eminence of its author, led to the uncritical acceptance of the myth. As Stephen Gould (who himself propagated this myth) wrote: "The most erroneous stories are those we think we know best-and therefore never scrutinize or question" (1996). It is hard to dissuade aging scientists, as they slip into their anecdotage, from repeating stories that they find amusing, but their younger colleagues must not mistake such stories for the history of science.

\section{ACKNOWLEDGMENTS}

We are grateful to Richard O'Connell, who introduced P. England to Perry's papers in 1983, to Stephen Johnston, Russell Pysklywec, and Norman Sleep for helpful comments, and to Craig Jones for correcting an error in Figure 1.

\section{REFERENCES CITED}

Burchfield, J.D., 1975, Lord Kelvin and the Age of the Earth: New York, Science History Publications, $260 \mathrm{p}$.

Carslaw, H.S., and Jaeger, J.C., 1959, Conduction of Heat in Solids, second ed.: Oxford, Oxford University Press, $510 \mathrm{p}$.

Chamberlin, T.C., 1899, Lord Kelvin's address on the age of the earth as an abode fitted for life: Science, v. 9, p. 889-901, doi: 10.1126/science.9.235.889.

Crosby, A.G., McKenzie, D., and Sclater, J.G., 2006, The relationship between depth, age, and gravity in the oceans: Geophysical Journal International, v. 66, p. 553-573.

Curie, P., and Laborde, A., 1903, Sur la chaleur dégagée spontanément par les sels de radium: Comptes Rendus de l'Académie des Sciences, v. 136, p. 673-675.

Dalrymple, G.B., 1991, The Age of the Earth: Stanford, Stanford University Press, 474 p.

Eve, A.S., 1939, Rutherford: Being the Life and Letters of the Rt. Hon. Lord Rutherford, O.M.: New York, Macmillan, 451 p.

Fourier, J.B.J., 1822, Théorie analytique de la chaleur: Paris, Académie des Sciences, p. 3,141 .

Fourier, J.B.J., 1827, Mémoire sur les températures du globe terrestre et des espaces planétaires: Mémoires de l'Académie Royale des Sciences de l'Institute de France, v. 7, p. 570-604.

Kelvin, W.T., 1863a, On the secular cooling of the earth: Transactions of the Royal Society of Edinburgh, v. 23, p. 157-170.

Kelvin, W.T., 1863b, On the rigidity of the earth; shiftings of the earth's instantaneous axis of rotation; and irregularities of the earth as a timekeeper: Philosophical Transactions of the Royal Society of London, v. 153, p. 573-582.

Kelvin, W.T, 1895, On the age of the earth: Nature, v. 51, p. 438-440.

Kelvin, W.T., 1899, The age of the earth as an abode fitted for life: Journal of the Transactions of the Victoria Institute, v. 31, p. 11-35.

Lindley, D., 2004, Degrees Kelvin: Washington, D.C., Joseph Henry Press, 366 p.

Lister, C.R.B., 1972, On the thermal balance of a mid-ocean ridge: Geophysical Journal of the Royal Astronomical Society, v. 26, p. 515-535.

Lyell, C., 1830, Principles of Geology, vol. 1: London, John Murray, 511 p.

McKenzie, D.P., Roberts, J.M., and Weiss, N.O., 1974, Convection in the earth's mantle: Towards a numerical simulation: Journal of Fluid Mechanics, v. 62, p. 465-538, doi: 10.1017/S0022112074000784.

Morton, J.L., and Sleep, N.H., 1985, A mid-ocean ridge model: Constraints on the volume of axial hydrothermal flux: Journal of Geophysical Research, v. 90, p. 11,345-11,354.

Oreskes, N., 1999, The rejection of Continental Drift: London, Oxford University Press, $420 \mathrm{p}$.

Parsons, B., and McKenzie, D.P., 1978, Mantle convection and the thermal structure of the plates: Journal of Geophysical Research, v. 83, p. 4485-4496.

Parsons, B., and Sclater, J.G., 1977, An analysis of the variation of ocean floor bathymetry and heat flow with age: Journal of Geophysical Research, v. 82, p. 802-827.

Perry, J., 1895a, On the age of the earth: Nature, v. 51, p. 224-227.

Perry, J., 1895b, On the age of the earth: Nature, v. 51, p. 341-342.

Perry, J., 1895c, On the age of the earth: Nature, v. 51, p. 582-585.

Richter, F.M. 1986, Kelvin and the age of the earth: Journal of Geology, v. 94, p. 395-401.

Sclater, J.G., Parsons, B., and Jaupart, C., 1981, Oceans and continents: Similarities and differences in mechanisms of heat loss: Journal of Geophysical Research, v. 86 , p. $11,535-11,552$

Stacey, F.D. 2000, Kelvin's age of the earth paradox revisited: Journal of Geophysical Research, v. 105, p. 13,155-13,158, doi: 10.1029/2000JB900028.

Turcotte, D.L., and Oxburgh, E.R., 1967, Finite amplitude convection cells and continental drift: Journal of Fluid Mechanics, v. 28, p. 29-42, doi: $10.1017 /$ S0022112067001880.

Turcotte, D.L., and Schubert, G., 2002, Geodynamics: Cambridge, Cambridge University Press.

Willis, B., 1942, American geology 1850-1900: Science, v. 96, no. 2486, p. 167-172, doi: 10.1126/science.96.2486.167

Manuscript received 9 August 2006; accepted 1 November

2006.

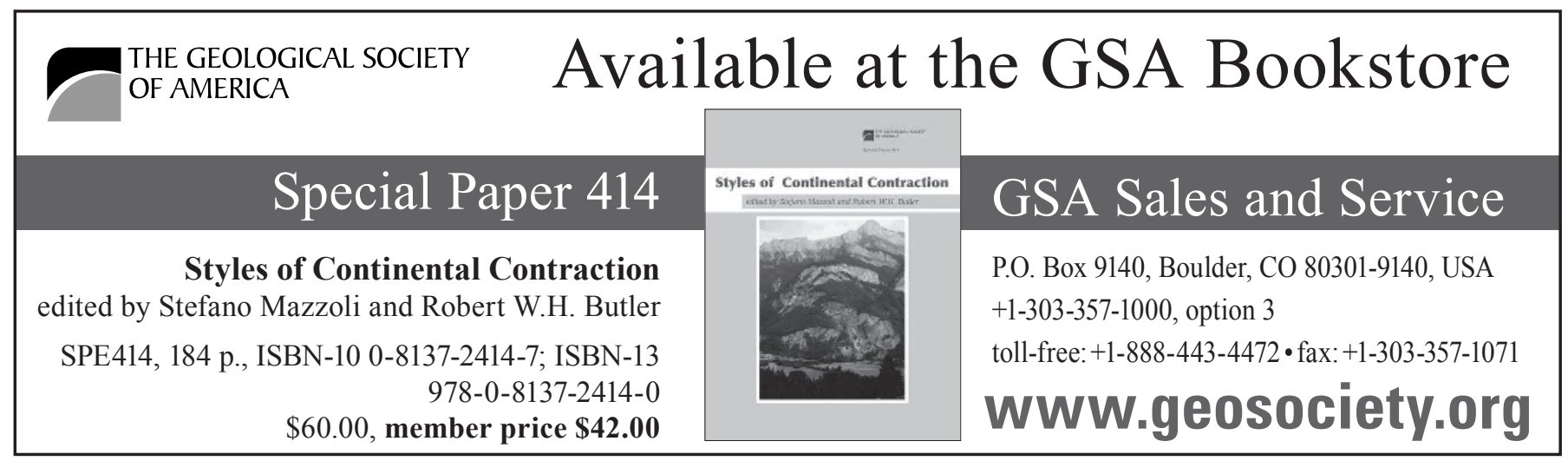

